\title{
VALUES OF NON-ATOMIC VECTOR MEASURE GAMES
}

\author{
Are They Linear Combinations of the Measures?*
}

\author{
Sergiu HART \\ Tel-Aviv University, 69978 Tel-Aviv, Israel \\ Abraham NEYMAN \\ The Hebrew University, 91904 Jerusalem, Israel
}

Submitled January 1986, accepted November 1987

\begin{abstract}
Consider non-atomic vector measure games; i.e., games $v$ of the form $v=f \circ\left(\mu_{1}, \ldots, \mu_{n}\right)$, where $\left(\mu_{1}, \ldots, \mu_{n}\right)$ is a vector of non-atomic non-negative measures and $f$ is a real-valued function defined on the range of $\left(\mu_{1}, \ldots, \mu_{n}\right)$. Games of this form arise, for example, from production models and from finite-type markets. We show that the value of such a game need not be a linear combination of the measures $\mu_{1}, \ldots, \mu_{n}$ (this is in contrast to all the values known to date). Moreover, this happens even for market games in $p N A$. In the economic models, this means that the value allocations are not necessarily generated by prices. All the examples we present are special cases of a new class of values.
\end{abstract}

\section{Introduction}

Much of economic theory is conccrned with the existence of prices. In particular, one is interested in whether various outcomes, defined by diverse postulates, turn out to be actually generated by prices. Whenever this is the case, a theory of endogenous price formation is obtained. In this paper we consider a well-known game-theoretic solution concept: the value. ${ }^{1}$ We will first describe the problem formally in game theoretic terms, and then we will present the corresponding class of economic models to which it applies.

Consider non-atomic games which are defined by finitely many nonnegative measures; i.e., games $v$ of the form $v=f \circ\left(\mu_{1}, \ldots, \mu_{n}\right)$, where $\left(\mu_{1}, \ldots, \mu_{n}\right)$ is a vector of non-atomic non-negative measures and $f$ is a realvalued function defined on the range of $\left(\mu_{1}, \ldots, \mu_{n}\right)$; a game of this form is

*The research was started at the Institute for Advanced Studies, the Hebrew University, Jerusalem, during the Research Year on Game Theory in 1979-1980, and was continued in summer 1985 at the University of California, Los Angeles. Financial support by National Science Foundations Grants SES-83-12190 and SES-85-10123 and by the U.S.-Israel Binational Science Foundation Grants $84-00201$ and $85-00342$ is gratefully acknowledged. We want to thank the editor, Andreu Mas-Colell, for useful suggestions on the presentation of these results.

${ }^{1}$ An alternative title for this paper might thus be 'Values of non-atomic economies: Are they generated by prices?'. 
usually called a vector measure game. It is a well-known fact that in all cases studied to date, the value of such a game $v$ turns out always to be a linear combination of the measures $\mu_{1}, \ldots, \mu_{n}{ }^{2}$

Can this 'fact' be proved in general? That is, is it a consequence of the axioms defining the value [cf. Aumann and Shapley (1974)]? We will show here that the answer is negative.

Formally, let $Q$ be a space of non-atomic games [all notations and definitions follow Aumann and Shapley (1974)], and let $\phi: Q \rightarrow F A$ be a value on $Q$. The property we discuss is

P.1. Let $\mu_{1}, \ldots, \mu_{n}$ be $n$ non-atomic non-negative measures, and $f$ a realvalued function defined on the range of $\left(\mu_{1}, \ldots, \mu_{n}\right)$. If $v=f \circ\left(\mu_{1}, \ldots, \mu_{n}\right)$ belongs to $Q$, then there exist real numbers $a_{1}, \ldots, a_{n}$ such that $\phi v=\sum_{i=1}^{n} a_{i} \mu_{i}$.

Note that P.1 holds when $n=1\left[\mathrm{by}^{3}\right.$ Proposition 6.1 in Aumann and Shapley (1974)]. We will construct a number of examples, each one consisting of a space of games $Q$ together with an appropriate value $\phi$ on it, such that P. 1 is violated. Of course, there might well be other values on the same space $Q$ which do satisfy P.1 (indeed, this is the case in all the examples in this paper).

Why is this question of interest? It turns out that in many applications, one usually encounters games of this form that depend on finitely many measures. Most notably, in mathematical economics, there are numerous such instances. We will consider here two standard examples.

The first one is a model of a production technology. There are $n$ production factors (inputs), out of which one final good (output) is produced, according to the function $f$. For each input $i$, let $x_{i}$ denote the quantity of input $i$, then $f\left(x_{1}, \ldots, x_{n}\right)$ is the quantity of output that may be produced from these inputs together. The ownership of each input $i$ is described by the measure $\mu_{i}$; i.e., a group of agents (a coalition) $S$ initially owns $\mu_{i}(S)$ units of input $i$. The total amount produced by $S$ is then precisely $v(S)=f\left(\mu_{1}(S), \ldots, \mu_{n}(S)\right)$. An outcome in this model is a certain payoff - a quantity of the final good that each agent receives. This may be described by a measure $\zeta$ : the total payoff of coalition $S$ is $\zeta(S)$. Assume that all the agents are insignificant (i.e., there is no single agent who initially owns a positive fraction of the total supply of some input; formally, this means that the measures $\mu_{i}$ are nonatomic). It is reasonable to expect in this case that prices for the inputs should arise. Thus, if we let $a_{i}$ be the price of input $i$ (i.e., 1 unit of input $i$ is worth $a_{i}$ units of output), then the outcome measure $\zeta$ is $a_{1} \mu_{1}+\cdots+a_{n} \mu_{n}$;

\footnotetext{
${ }^{2}$ This is also true for other solution concepts; e.g., the core - see Billera and Raanan (1981).

${ }^{3}$ Here and in the sequel we always assume that the underlying measurable space $(I, C)$ is standard (i.e., isomorphic to $([0,1], \boldsymbol{B})$, where $\boldsymbol{B}$ denotes the Borel $\sigma$-field).
} 
and, conversely, if $\zeta$ is such a linear combination of the $\mu_{i}$ 's then the corresponding coefficients $a_{i}$ may be interpreted as input prices. Property P.1 can therefore be translated in this model as follows: to every value outcome there correspond prices for the production factors. As we have stated above, it turns out that this is, however, not true in general.

A second model is that of a market (exchange economy) with transferable utility [cf. Aumann and Shapley (1974, ch. VI)]: the space of agents is $I$, and $\mu$ is the population measure. There are $l$ goods. Each agent $t$ in $l$ has an initial endowment ${ }^{4} e(t) \in \mathbb{R}_{+}^{l}$ and a concave function $u_{t}: \mathbb{R}_{+}^{l} \rightarrow \mathbb{R}$, which may be interpreted either as a utility function or as a production function. Assume that there are only finitely many different functions $u_{t}$; i.e., the space of agents $I$ is partitioned into disjoint sets $T_{1}, \ldots, T_{k}$, such that $u_{t}=U_{j}$ for all $t \in T_{j}$ and $j=1, \ldots, k$. This is called the finite type case (note that 'type' refers here only to the utility functions and not to the initial endowments). Put $n=k+l$, and define the function $f: \mathbb{R}_{+}^{n} \rightarrow \mathbb{R}$ by

$$
f\left(y_{1}, \ldots, y_{k} ; z_{1}, \ldots, z_{l}\right)=\max \left\{\sum_{j=1}^{k} y_{j} U_{j}\left(x_{j}\right) \mid x_{j} \in \mathbb{R}_{+}^{l}, \sum_{j=1}^{k} y_{j} x_{j} \leqq\left(z_{1}, \ldots, z_{l}\right)\right\} .
$$

Then the maximum that is achieveable by a coalition $S$ equals [see Aumann and Shapley $(1974,(39.18))]$

$$
v(S)=f\left(\eta_{1}(S), \ldots, \eta_{k}(S) ; \zeta_{1}(S), \ldots, \zeta_{l}(S)\right),
$$

where $\eta_{j}(S)=\mu\left(S \cap T_{j}\right.$ ) (the proportion of type $j$ in $S$ ) and $\zeta_{i}(S)=\int_{S} e_{i} \mathrm{~d} \mu$ (the total initial endowment of $S$ of good $i)$. Note that the measures $\eta_{j}$ are mutually singular, whereas the measures $\zeta_{i}$ need not be so. In this setup, a linear combination of the measures, $\sum_{j} a_{j} \eta_{j}+\sum_{i} b_{i} \zeta_{i}$, corresponds to a price $b_{i}$ per unit of good $i$, and a price $a_{j}$ per type $j .^{5}$

Since we will show that a value need not satisfy P.1, it follows that the classical axiomatic approach to the value in the non-atomic case [cf. Aumann and Shapley (1974)] does not yield the existence of prices for the economic factors in the corresponding models. It should therefore be of interest to find, on one hand, additional plausible axioms that would imply P.1, and, on the other hand, reasonable economic interpretations to the values we exhibit here - where P.1 is violated and there are thus no underlying prices.

\footnotetext{
${ }^{4} \mathbb{R}$ denotes the real line, and $\mathbb{R}_{+}^{l}$ is the non-negative orthant of the $l$-dimensional Euclidean space.

${ }^{s}$ The two models are actually quite similar: on one hand, a finite type market with one type $(k=1)$ corresponds to a production technology. On the other hand, if one considers the $k$ types as additional 'goods', then the function $f$ defined above represents a production technology with $n=k+l$ inputs.
} 


\section{The examples}

We start with a simple counter-example to P.1.

Example 1. Let $\mu_{1}$ and $\mu_{2}$ be two non-atomic probability measures on $(I, C)=$ $([0,1], \boldsymbol{B})$, and consider the game $v=\min \left\{\mu_{1}, \mu_{2}\right\}$. For each $i=1,2$ let $g_{i}=$ $\mathrm{d} \mu_{i} / \mathrm{d} \mu$ be the Radon-Nikodym derivative of $\mu_{i}$ with respect to $\mu=\mu_{1}+\mu_{2}$. Define $\zeta=\mu_{1} \wedge \mu_{2}$ by $\mathrm{d} \zeta / \mathrm{d} \mu=\min \left\{g_{1}, g_{2}\right\}$. We now require that $\zeta$ is not a linear combination of $\mu_{1}$ and $\mu_{2}$; in particular, $\zeta$ does not vanish. For example, let $\lambda$ be the Lebesgue measure on $([0,1], \boldsymbol{B}), \mu_{1}=\lambda$ and $\mu_{2}(S)=$ $\int_{s} 2 s \mathrm{~d} \lambda(s)$; then $\zeta(S)=\int_{s} \min \{2 s, 1\} \mathrm{d} \lambda(s)$ is not a linear combination of $\mu_{1}$ and $\mu_{2}$ [indeed, $(\mathrm{d} \zeta / \mathrm{d} \lambda)(s)$ is not an affine function of $s$, whereas $\left(\mathrm{d}\left(a_{1} \mu_{1}+\right.\right.$ $\left.\left.a_{2} \mu_{2}\right) / \mathrm{d} \lambda\right)(\mathrm{s})$ is so for all real $\left.a_{1}, a_{2}\right]$.

Let $Q$ be the symmetric linear space generated by the game $v$; an element $w$ of $Q$ is thus of the form

$$
w=\sum_{i=1}^{n} a_{i} \theta_{i}^{*} v
$$

where, for each $i=1, \ldots, n, a_{i}$ is a real number, $\theta_{i}$ is an automorphism of the measurable space $(I, C)$, and $\theta_{i}^{*}$ denotes the induced symmetry on games: $\left(\theta_{i}^{*} v\right)(S)=v(\theta S)$ for all $S \in C$. Define an operator $\psi: Q \rightarrow F A$ by

$$
\psi\left(\sum_{i=1}^{n} a_{i} \theta_{i}^{*} v\right)=\sum_{i=1}^{n} a_{i} \theta_{i}^{*} \zeta
$$

We claim that $\psi$ is well-defined (i.e., $\psi w$ does not depend on the particular reprcsentation of $w$ ), that it is symmetric, linear and positive. As usual [see, e.g., Proposition 4.1 in Neyman and Tauman (1979)], it suffices to prove positivity; the rest then follows easily. To this end, let $I_{1}=\left\{s \in I \mid g_{1}(s)<g_{2}(s)\right\}$ and $I_{2}=I \backslash I_{1}$; note that

$$
v(S)=\zeta(S)
$$

for all $S \in C$ such that either $S \subset I_{1}$ or $S \subset I_{2}$. Therefore, if $S \in C$ satisfies $S \subset \theta_{i}^{-1} I_{1}$ or $S \subset \theta_{i}^{-1} I_{2}$ for all $i=1, \ldots, n$, then

$$
w(S)=\sum_{i=1}^{n} a_{i} v\left(\theta_{i} S\right)=\sum_{i=1}^{n} a_{i} \zeta\left(\theta_{i} S\right)=(\psi w)(S) .
$$

Let $w$ be a monotonic game; we thus have $(\psi w)(S)=w(S) \geqq 0$ for all coalitions $S$ as above. Since $\psi w$ is a measure, and every coalition $T \in C$ is a union of such sets $S$, it follows that $\psi w \geqq 0$, i.e., $\psi$ is a positive operator. ${ }^{6}$

${ }^{6} \mathrm{~A}$ similar argument was used in Tauman (1982, Lemma 2). 
To obtain a value $\phi$ from $\psi$, we only need to normalize it, so that efficiency is also satisfied:

$$
\phi w=\frac{1}{\zeta(I)} \psi w
$$

This completes Example 1: $\phi$ is a value on $Q$ that does not satisfy P.1.

How 'exceptional' is this example? We will show that one may actually require additional conditions, buth on the space $Q$ and on the value $\phi$, and still obtain a counter-example to P.1.

Recall that a value is defined by the following axioms: additivity, symmetry, positivity and efficiency. Another desirable property is the

Projection Axiom. $\phi \mu=\mu$ for all finitely additive measures $\mu^{7}$

Of course, this condition applies only when $\mu$ belongs to the space on which the operator $\phi$ is defined. The space $Q$ of Example 1, however, does not contain any positive finitely additive measure. ${ }^{8}$ An (indirect) argument showing that no positive finitely additive measure belongs to $Q$ is as follows: let $\mu$ be such a measure in $Q$, then $\mu$ is absolutely continuous (since the generating game $v$ is absolutely continuous), and thus $\mu$ is a non-atomic measure. By symmetry and linearity, $Q$ must therefore contain all positive non-atomic measures, in particular $\zeta$. Now

$$
v-\zeta=\min \left\{\mu_{1}-\zeta, \mu_{2}-\zeta\right\}
$$

The measures $\mu_{1}-\zeta$ and $\mu_{2}-\zeta$ are easily seen to be mutually singular positive non-atomic measures, therefore any value $\phi$ satisfies [by the same argument as that of Proposition 19.7 in Aumann and Shapley (1974)]

$$
\phi\left(\min \left\{\mu_{1}-\zeta, \mu_{2}-\zeta\right\}\right)=\frac{1}{2}\left(\mu_{1}-\zeta\right)+\frac{1}{2}\left(\mu_{2}-\zeta\right)=\frac{1}{2}\left(\mu_{1}+\mu_{2}\right)-\zeta
$$

This implies that $\phi v=\frac{1}{2}\left(\mu_{1}+\mu_{2}\right)$.

The argument above shows not only that $Q$ does not contain any positive measure, but, moreover, that the value $\phi$ on $Q$ cannot be extended to any larger space that will include $N A$, the space of all non-atomic measures.

Our examples below will be of values that satisfy the Projection Axiom, on spaces that will contain $N A$.

\footnotetext{
${ }^{7}$ Note that if $\mu$ is a non-atomic countably additive measure, then $\phi \mu=\mu$ is a consequence of symmetry and efficiency.

${ }^{8}$ It contains all non-atomic countably additive measures with total mass zero; indeed, let $\theta(s)=1-s$ for all $s \in[0,1]$, then $v-\theta^{*} v=\mu_{2}-\mu_{1}$.
} 
We next consider conditions on the space $Q$. In both Examples 2 and 3 below, we show that $Q$ can be made large enough so as to contain the 'differentiable' games: $p N A$, or even $D I F F$ [see Mertens (1980)]. Moreover, the value $\phi$ will of course coincide on $p N A$ with the unique value there, and on $D I F F$ it will coincide with Mertens' value $\phi_{D}$.

Example 2 is obtained by a slight modification of Example 1; it has, however, the drawback that the game $v$ where P.1 is violated is not monotonic. This is remedied in Example 3, where $v$ is a market game [the minimum of three measures; of course, not mutually singular - see Proposition 19.7 in Aumann and Shapley (1974)].

Finally, we will also show that $Q$ may be taken to consist only of differentiable games. In Example $4, Q$ is the space generated by a market game in $p N A$, or, alternatively, it is a subspace of $p N A$ containing $N A$.

We now present these examples. They will be followed in section 3 by the general construction of a new class of values, of which all our examples are special cases. We hope that these values may well be of independent interest.

Example 2. Let $(I, C)=([0,2], B)$, and let $\mu_{1}$ and $\mu_{2}$ be as in Example 1 (their support is $[0,1]$ ). Let $\mu_{3}$ and $\mu_{4}$ be the measures with support $[1,2]$ obtained by translating $\mu_{1}$ and $\mu_{2}$, respectively: for $i=1,2, \mu_{i+2}(S)=\mu_{i}(S-1)$, where $S-1=\{s-1 \mid s \in S\}$ for $S \subset[1,2]$. Let $\zeta=\mu_{1} \wedge \mu_{2}$ and $\zeta^{\prime}=\mu_{3} \wedge \mu_{4}$ be defined as in Example 1. Finally, let

$$
v=\min \left\{\mu_{1}, \mu_{2}\right\}-\min \left\{\mu_{3}, \mu_{4}\right\}
$$

and define $Q$ as the linear symmetric space $^{9}$ generated by $v$ and $N A$. An element $w$ of $Q$ is thus of the form

$$
w=\sum_{i=1}^{n} a_{i} \theta_{i}^{*} v+\eta
$$

where $\eta \in N A$ and $a_{i}, \theta_{i}$, are as before. The operator $\phi: Q \rightarrow F A$ is defined by

$$
\phi\left(\sum_{i=1}^{n} a_{i} \theta_{i}^{*} v+\eta\right)=\sum_{i=1}^{n} a_{i} \theta_{i}^{*}\left(\zeta-\zeta^{\prime}\right)+\eta
$$

Note that $\phi$ is efficient [since $\left.v(I)=0=\zeta(I)=\zeta^{\prime}(I)\right]$, and satisfies all the axioms of a value (use the same proof as in Example 1).

\footnotetext{
${ }^{9}$ Note that $Q$ is a reproducing space: $v=\left(v+\mu_{3}+\mu_{4}\right)-\left(\mu_{3}+\mu_{4}\right)$ is a difference of two monotonic games.
} 
Actually, one may add to $Q$ also $p N A$ and even $D I F F$. Indeed, let

$$
w=\sum_{i=1}^{n} a_{i} \theta_{i}^{*} v+u
$$

with $u$ a game in DIFF. Assume $w$ is monotonic; then its extension $\bar{w}$ is also monotonic, therefore

$$
\frac{1}{\tau}[\bar{w}(t I+\tau S)-\bar{w}(t I)] \geqq 0
$$

for all $S \in C, t \in(0,1)$ and $\tau>0$ sufficiently small. It is easily seen that the above expression equals

$$
\sum_{i=1}^{n} a_{i} \theta_{i}^{*} v(S)+\frac{1}{\tau}[\bar{u}(t I+\tau S)-\bar{u}(t l)]
$$

Integrate over $t$ in $(0,1)$ and the take the limit as $\tau \rightarrow 0^{+}$, to obtain

$$
\sum_{i=1}^{n} a_{i} \theta_{i}^{*} v(S)+\left(\phi_{D} u\right)(S) \geqq 0
$$

(where $\phi_{D}$ denotes Mcrtens' value on $D I F F$ ).

We again consider first coalitions $S$ for which

$$
\theta_{i}^{*} \min \left\{\mu_{1}, \mu_{2}\right\}(S)=\theta_{i}^{*} \zeta(S) \text { and } \theta_{i}^{*} \min \left\{\mu_{3}, \mu_{4}\right\}(S)=\theta_{i}^{*} \zeta^{\prime}(S)
$$

for all $i$. Then $\theta_{i}^{*} v(S)=\theta_{i}^{*}(\phi v)(S)$, implying $(\phi w)(S) \geqq 0$. The proof is completed by decomposing any $T \in C$ into a union of such coalitions $S$.

Example 3. Let $\mu_{1}, \mu_{2}$ and $\mu_{3}$ be three non-atomic probability measures (not mutually singular). Put

$$
\begin{aligned}
& v=\min \left\{\mu_{1}, \mu_{2}, \mu_{3}\right\}, \quad \mu=\mu_{1}+\mu_{2}+\mu_{3}, \\
& \bar{\mu}=\mu_{1} \vee \mu_{2} \vee \mu_{3}, \text { and } \underline{\mu}=\mu_{1} \wedge \mu_{2} \wedge \mu_{3}
\end{aligned}
$$

(i.e., $\mathrm{d} \bar{\mu} / \mathrm{d} \mu=\max _{1 \leqq i \leqq 3}\left\{\mathrm{~d} \mu_{i} / \mathrm{d} \mu\right\}$ and $\mathrm{d} \underline{\mu}=\min _{1 \leqq i \leqq 3}\left\{\mathrm{~d} \mu_{i} / \mathrm{d} \mu\right\}$ ). Choose the real constant $a$ so that

$$
\zeta=a \underline{\mu}+(1-a) \bar{\mu}
$$


is a probability measure [i.e., $\zeta(I)=1$; note that $0 \leqq \mu(I) \leqq 1 \leqq \bar{\mu}(I) \leqq \mu(I)$, thus $0 \leqq a \leqq 1]$. We now require that $\zeta$ is not a linear combination of the $\mu_{i}$ 's.

For example, let $g$ be the following real function on $[0,1): g(s)=0$ for $s \in\left[0, \frac{1}{3}\right) ; g(s)=2 s$ for $s \in\left[\frac{1}{3}, \frac{2}{3}\right)$; and $g(s)=2$ for $s \in\left[\frac{2}{3}, 1\right)$. Let $\lambda$ be the Lebesgue measure on $(I, C)=([0,1], B)$ and put $\left(\mathrm{d} \mu_{i} / \mathrm{d} \lambda\right)(s)=g([s+(i-1) / 3][\bmod 1])$ for $i=1,2,3$. Then $\bar{\mu}=2 \lambda, \underline{\mu}=0$ and $\zeta=\lambda$ (which is indeed not a linear combination of the $\mu_{i}$ 's).

Let $Q$ be the symmetric linear space generated by $v$ together with $D I F F$, and let $\phi$ be the symmetric linear operator on $Q$ defined by $\phi v=\zeta$ and $\phi w=\phi_{D} w$ for all $w \in D I F F$ (recall that $\phi_{D}$ denotes Mertens' value on DIFF). It may be checked that $\phi$ is a value on $Q$ (of course, P.1 is not satisfied). This will follow in particular from the general arguments below.

Example 4. Let $\mu_{1}$ and $\mu_{2}$ be two non-atomic probability measures that are not mutually singular, and let $v=\sqrt{\mu_{1} \cdot \mu_{2}}$. Then $v$ is a market game ${ }^{10}$ in $p N A$ [indeed, $\sqrt{\mu_{i}} \in p N A$ by Theorem $C$ in Aumann and Shapley (1974), and $p N A$ is an algebra]. Let

$$
\psi v(S)=\int_{S} \sqrt{\frac{\mathrm{d} \mu_{1}}{\mathrm{~d} \mu} \cdot \frac{\mathrm{d} \mu_{2}}{\mathrm{~d} \mu}} \mathrm{d} \mu,
$$

where $\mu=\mu_{1}+\mu_{2}$ and $S \in C$. For an appropriate constant $a$, the operator $a \psi$ can be extended to a value on the linear symmetric space generated by $v$.

Moreover, if $\theta$ is an automorphism such that $v$ and $\theta^{*} v$ have disjoint carriers, then $\psi$ itself (without the constant $a$ ) is a value on the space generated by $u=v-\theta^{*} v$, which can be extended to a value on the space generated by $u$ together with $N A$.

Remark. The last example shows, in particular, that on subspaces of $p N A$ the value need not be unique. ${ }^{11}$

\section{A class of values}

We come now to the general construction of a new class of values, of which all the four examples above are special cases. We hope that these values may be of independent interest and find other applications as well.

Let $Q$ be a linear symmetric space of games, i.e., functions $v: C \rightarrow \mathbb{R}$ with $v(\varnothing)=0$. Let $v \rightarrow \bar{v}$ be an (extension) operator that associates to every game $v$

\footnotetext{
${ }^{10}$ I.e., super-additive, monotonic and positively homogeneous of degree one.

"Another example: for every $v \in p N A$, let $\psi v=2 \int_{0}^{1 / 2} \partial \bar{v}(t, S) \mathrm{d} t$. Then $\psi$ is a continuous value (of norm 2) on the symmetric linear subspace $Q$ of $p N A$ on which it is efficient (i.e., $v \in Q$ if and only if $v(I)=\psi v(I) \equiv 2 v\left(\frac{1}{2} I\right)$; note that $Q$ includes all homogeneous games in $p N A$, in particular $\left.N A\right)$.
} 
in $Q$ a real valued function $\vec{v}$ defined on simple functions $h$ which are measurable with respect to $C$, satisfying:

(i) $\bar{v}(h)=\bar{v}((h \vee 0) \wedge 1) ; \bar{v}(0)=0 ; \bar{v}(1)=v(I)$; and $\bar{v}$ is continuous at 0 and 1 ;

(ii) if $v$ is monotonic then $\bar{v}$ is monotonic;

(iii) if $v \in F A \cap Q$ and $0 \leqq h \leqq 1$, then $\bar{v}(h)=\int_{S} h \mathrm{~d} v$;

(iv) If $\theta$ is an automorphism of $(I, C)$, then $v \circ \theta=\bar{v} \circ \theta$, where $(\theta h)(s)=h(\theta s)$;

(v) the operator $v \rightarrow \bar{v}$ is linear.

For any pair $\lambda, \eta$ of measures on $[0,1)$ and $(0,1]$, respectively, any finite subfield $\Pi$ of $C$ and any $\tau>0$, let $\psi(\lambda, \eta ; \Pi ; \tau) v$ be the measure on $(I, \Pi)$ defined by

$$
\begin{aligned}
{[\psi(\lambda, \eta ; \Pi ; \tau) v](S)=} & \frac{1}{\tau} \int_{0}^{1}[\bar{v}(t I+\tau S)-\bar{v}(t I)] \mathrm{d} \lambda(t) \\
& +\frac{1}{\tau} \int_{0}^{1}[\bar{v}(t I)-\bar{v}(t I-\tau S)] \mathrm{d} \eta(t),
\end{aligned}
$$

for every atom $S$ of $\Pi$, whenever all the integrals exist. Next, define the measure $\psi(\lambda, \eta ; \Pi) v$ on $(I, \Pi)$ as the limit of measures $\psi(\lambda, \eta ; \Pi ; \tau) v$ as $\tau \rightarrow 0^{+}$, whenever the limit exists. Finally, we define $\psi(\lambda, \eta) v$ on $(I, C)$ as the limit again, whenever it exists - of the measures $\psi(\lambda, \eta ; \Pi) v$ as $\Pi$ increases (i.e., for every $S \in C$ and every $\varepsilon>0$ there exists a finite field $\Pi$ with $S \in \Pi$ such that for every finite field $\Pi_{1}$ that includes $\Pi$,

$$
\left|\left[\psi\left(\lambda, \eta ; \Pi_{1}\right) v\right](S)-[\psi(\lambda, \eta ; \Pi) v](S)\right|<\varepsilon .
$$

Let $Q(\lambda, \eta)$ be the set of all games $v$ in $Q$ for which $\psi(\lambda, \eta) v$ exists and is finite (that is, all the above integrals and limits exist and are finite). It is now easy to check the following:

(a) $Q(\lambda, \eta)$ is a linear symmetric subspace of $Q$.

(b) $\psi(\lambda, \eta)$ is a linear and symmetric operator from $Q(\lambda, \eta)$ into $F A$.

(c) If both $\lambda$ and $\eta$ are non-negative measures, then $\psi(\lambda, \eta)$ is a positive operator.

(d) If both $\lambda$ and $\eta$ are non-negative measures, then $\psi(\lambda, \eta)$ is a value operator on the linear symmetric subspace $Q^{\mathfrak{e}}(\lambda, \eta)$ of $Q(\lambda, \eta)$ on which it is efficient [i.e., $Q^{\mathrm{e}}(\lambda, \eta)$ consists of all $v \in Q(\lambda, \eta)$ for which $\left.[\psi(\lambda, \eta) v](I)=v(I)\right]$.

(e) If $\lambda([0,1))+\eta((0,1])=1$ then $\psi(\lambda, \eta)$ satisfies the Projection Axiom and, moreover, $Q^{\mathfrak{e}}(\lambda, \eta) \supset F A \cap Q$; otherwise, $Q^{\mathfrak{e}}(\lambda, \eta) \cap F A^{+}=\{0\}$.

All the values in our examples are obtained as $\psi(\lambda, \eta)$ for a pair of non- 
negative measures $\lambda, \eta$. We always use the standard extension operator, $Q$ being a subspace of $p N A^{\prime}$ or $E X T$ [see Mertens (1980)].

In example $1, \eta=0$ and $\lambda$ is any measure on $[0,1)$ of total mass $1 / \zeta(I)$ [note that when $v=\min \left\{\mu_{1}, \mu_{2}\right\}$ we have $\bar{v}(t I+\tau S)-\bar{v}(t I)=\bar{v}(\tau S)$ and therefore $\psi(\lambda, 0) v$ depends only on the total mass of $\lambda]$. In Example 2, again $\eta=0$, but now $\lambda([0,1))=1$.

We come next to Example 3. If $v \in Q$ has a concave extension $\bar{v}$, and the marginals of $\bar{v}$ in a neighborhood of the diagonal are bounded by the marginals of some measure, then $v \in Q(\lambda, 0)$ and $v \in Q(0, \lambda)$ for any measure $\lambda$. Indeed, the concavity of $\bar{v}$ and the monotone convergence theorem yield $[\psi(\lambda, 0 ; \Pi) v](S)=\int_{0}^{1} \partial \bar{v}(t, S) \mathrm{d} \lambda(t)$ for all atoms $S$ of $\Pi$; this expression increases with $\Pi$ (again by the concavity of $\vec{v}$ ) and is bounded from above (by our assumption on the marginals of $\vec{v}$; this implies that $v \in Q(\lambda, 0)$. Similarly we obtain $v \in Q(0, \lambda)$, implying also $v \in Q(a \lambda,(1-a) \lambda)$ for all $0 \leqq a \leqq 1$.

If $\lambda$ is the Lebesgue measure, then in addition we have the following: $[\psi(\lambda, 0 ; \Pi) v](I) \leqq v(I) \leqq[\psi(0, \lambda ; \Pi) v](I)$ (recall the representation as an integral above), hence also $[\psi(\lambda, 0) v](I) \leqq v(I) \leqq[\psi(0, \lambda) v](I)$. Therefore $v \in Q^{\mathrm{e}}(a \lambda,(1-a) \lambda)$ for an appropriate constant $a \in[0,1]$. Finally, note that $\psi(a \lambda,(1-a) \lambda)$ coincides on $Q \cap D I F F$ with $\phi_{D}$ (for any $a \in[0,1]$ ).

In Example 4 we consider $\psi(\lambda, 0)$ with $\lambda$ an atomic measure concentrated at the point 0 : on the space generated by $v=\sqrt{\mu_{1} \cdot \mu_{2}}$ there, $\lambda(\{0\})=a$, and on the space generated by $u=v-\theta^{*} v$ and $N A, \lambda(\{0\})=1$.

\section{References}

Aumann, R.J. and L.S. Shapley, 1974, Values of non-atomic games (Princeton University Press, Princeton, NJ).

Billera, L.J. and J. Raanan, 1981, Cores of nonatomic linear production games, Mathematics of Operations Research 6, 420-423.

Mertens, J.-F., 1980, Values and derivatives, Mathematics of Operations Research 5, 523-552.

Neyman, A. and Y. Tauman, 1979, The partition value, Mathematics of Operations Research 4, 236-264.

Tauman, Y., 1982, Value on a class of non-differentiable market games, International Journal of Game Theory $10,155-162$. 